\title{
Response of some white Corn (Zea mays $L$.) hybrids to Bio-mineral fertilizer application under AL-Frafra Oasis conditions.
}

\author{
Abd El-Lateef A. A. and A.M. Bughdady \\ Plant Production Dept., Desert Research Center, El-Matareya, Cairo, Egypt.
}

\begin{abstract}
Yield and quality of white corn may be improved by manipulating the cultural practices to suit the crop with the prevailing environment. Therefore, two field experiments were carried out at AL-Frafra Oasis, New Valley Governorate, Egypt, in sandy clay loam soil, during the early summer seasons 2014 and 2015 growing seasons, to study the response of three corn hybrids of white corn and eightbio-mineral fertilizers treatments (a mixture of bio-fertilizer Rizobium and Aztobacter) and the percentages of recommended mineral fertilizer in the region as combination from mineral NPK on yield, it components and chemical contents on grains of white corn hybrids.

Planting Single hybrid $30 \mathrm{~K} 8(\mathrm{SH} 30 \mathrm{k} 8)$ caused an increase in all yield, and its components and grain chemical composition such as plant height $(\mathrm{cm})$, ear length $(\mathrm{cm})$, ear diameters $(\mathrm{cm})$, no. of grains/ear, 100grains weight (g), grain and biological yields ( $\mathrm{kg} / \mathrm{fed}), \mathrm{N}(\%), \mathrm{P}(\%), \mathrm{K}(\%)$ and crud protein content $(\%)$ in both Seasons except no. of ear/plant, stover yield and total carbohydrate (\%)in first season and $\mathrm{P}(\%)$ in the second season. Results indicated that, adding F6 treatment led to significantly increased all parameters in both seasons, except no. of ear/plant, stover, crud protein and total carbohydrate in the first season and100 grains weight, grain yield, crud protein content and total carbohydrate (\%) in the second season were significantly increased by adding F2 treatment. The interaction between white corn hybrids and Fertilizers on the yield, its components and grain chemical content. Results revealed that maximum criteria were obtained by (SH30k8) and F6 in first season. Similarly, results were obtained in second season except plant height and total carbohydrate were obtained by (SH30k8) and F2.
\end{abstract}

Key words: White corn, hybrids, bio- mineral fertilizer, Yield, chemical composition, AL-Frafra Oasis.

\section{Introduction}

Corn (Zea mays L.) is one of the most important cereal crops and consumed all over the world; because of its high diversity in form, quality and growth habit in a wide part of regions prone to agriculture of world is planted and utilized. while grain yields of the different Maize (Zea mays L.) is the third most important cereal crops in the world after wheat and rice and known as "King of grain crops Tollenaar and Dwyer, (1999). High yield of dry corn substances and grain, diverse nutritional value to supply carbohydrate, crude protein and edible oil and also high efficiency of water application in agricultural economy in different nations is particular important Choudhary andKumar,( 2013). Furthermore, NPK are considered of the major nutrients of growth for plant.

This nutrient is a basis of formation of crud protein and nucleic acid and supply of its required amount is very necessary for plant. NPK are applied as chemical fertilizer and its supply by this way is one of causes of water cycle pollution in nature, as well as, environmental pollution, also, production of them is very expensive. Thus, the partial replacement of recommended nitrogen fertilizer by bio-fertilizers is frequently recommended firstly for improve biological, physical and chemical properties of soil and secondly to get clean agricultural products free of undesirable high doses of heavy metals and other environmental pollutants. Generally, our present scientific work was applied to study response of three white maize hybrids to partial replacement of recommended nitrogen fertilizer by Bio-fertilizers. Bio-fertilizer usually contains microorganisms having specific function such as Aztobacter to fix nitrogen and $\mathrm{P}$ solubilizing bacteria to solubilize $\mathrm{P}$ from the soil and fertilizer to be available to the plants. Several researchers had conducted the experiments to evaluate the responses of various plants such as corn to the bio-fertilizer application.

Application of bio-fertilizers became of great necessity to get a yield of high quality and to avoid the environmental pollution, for gave to highest seed yield in agriculture addition to both nitrogen and phosphate fertilizer is very important. Azimiet al.,(2013) found that application of bio-fertilizer gave the highest seed yield (7.6 ton/ha) and nonapplication of bio-fertilizers treatment has the lowest seed yield (6.3 ton/ha). Also they suggested that grain yield and biomass yield increasing was reported with the bio-fertilizer application which account important benefit, causing decreasing in the inputs of production because of economizing much money to chemical fertilizers.

In maize, application of bio-fertilizers increased yield in many researches. Increased root, shoot weight with dual inoculation in maize have been reported by(Ahmed 2009). Maize hybrids differed in its productivity as well as its response to nitrogen fertilization, also, growth parameters were affected by maize hybrids (Chaudhryet al., 2005 and Sharifi 
and Taghizadeh 2009). Nitrogen fertilizer greatly effect on vegetative growth, maize yield as well as grain quality (Ahmed, 1998). Nitrogen application had pronounced effect in increasing vegetative growth of crop plants (Khan et al., 1999). Chemical constituents of corn grains as carbohydrate and oil concentrations are significant increase by nitrogen application (Ibrahim and Kandil, 2007) also, enhanced the amino acid formation and proline content (Ali et al., 1999). Nitrogen application improved maize yield (Mohamed et al.,2000).

\section{Materials and Methods}

Two field experiments were carried out during two successive summer seasons 2014 and 2015 at AL-Frafra Oasis, New Valley Governorate, and Egypt. The Textural class of soil of the site is sandy clay loam containing $1.48 \%$ and 1.69 for organic matter, 8.48 and8.44 pH , 2.21 and $2.28 \mathrm{dS} / \mathrm{m} \mathrm{EC}$ in the first and second seasons respectively. The study included two tested factors, i.e. white corn (Zea mays L.) hybrids and bio-mineral fertilization. Biomineral fertilizer treatments (Rezobium and Aztobacter) and percentages of recommended mineral fertilizer in the region as combination from mineral NPK were added as flowed: . F1 without (control), F2(100\% mineral NPK), F3(Rezobium + $75 \%$ NPK), F4(Rezobium+50 \% NPK), F5(Rezobium + \% 25 NPK ), F6 (Aztobacter $+75 \%$ NPK), F7 (Aztobacter $+50 \quad \% \quad$ NPK) and F8(Aztobacter $+25 \%$ NPK) and corn hybrids, three corn hybrids $(\mathrm{YCH})$ i.e. (High Tech Hybrid(HTH), (Single hybrid $30 \mathrm{~K} 8(\mathrm{SH} 30 \mathrm{k} 8)$ and (Triangle Hybrid(TH) were sown as early summer on 3 and7 of May in first and second seasons with gated pipe irrigation system, well water as the source of irrigation with $\mathrm{pH}$ of 7.00 and $\mathrm{EC}$ of $1.08 \mathrm{dS} / \mathrm{m}$, was used. irrigation were added as a recommendation in region every 6-8 days.

A split-plot design with three replicates was used. The corn hybrids treatments were arranged in the main plots and fertilization were distributed in the sub plots. The experimental unit area was $10.5 \mathrm{~m} 2$ (3 $\mathrm{m}$ length $\times 3.5 \mathrm{~m}$ width) containing four ridges with $0.75 \mathrm{~m}$ in width, the distance pen hills $25 \mathrm{~cm}$ with one plant for hill.

The recommended in the region ( $250 \mathrm{~kg}$ calcium super phosphate / fed. (15.5\% P2O5) mixed with the surface layer. In addition, $200 \mathrm{~kg}$ ammonium phosphate / fed. $(20.5 \% \mathrm{~N})$ and $200 \mathrm{~kg}$ potassium phosphate / fed.(48\% K2O) were applied at three doses The first dose was added at 21days from sowing, directly before thinning, and the second one at the 35 days after sowing and the third at 50 days from sowing. Bio- fertilization was mixed with grain at sowing.

At harvest, ten guarded plants were randomly taken from each plot to measure plant height $(\mathrm{cm}$.), ear length and diameter $(\mathrm{cm}$.), no. of ear /plant, no. of grains/ear, 100- grain weight ( $\mathrm{g}$ ), grain yield ( $\mathrm{kg}$ /fed), stover yield and biological yield (kg/fed). $\mathrm{N}(\%), \quad \mathrm{P}(\%), \quad \mathrm{K}(\%)$, crud protein content $(\%)$ and total carbohydrate ( \%). Grains nitrogen content, total nitrogen was determined using modified microKjeldahl method as described by (Peach and Tracey 1956). The crud protein content was calculated by multiply by 5.7 to obtain the crud protein percentage.), $\mathrm{P}$, Kcontent percentage was determined photo-metrically using flame photometer model concerning as described by(Johnson and Ulrich 1959)., total carbohydrates \% (total available carbohydrates were extracted according to (Smith $\boldsymbol{e t}$. al., 1964) and estimated calorimetrically by phenolsulphuric acid method as described by (Montogomery, 1961).

All the obtained data were exposed to the proper statistical analysis of variance according to (Gomez and Gomez,1984).in three replicates, For comparison between treatments, the least significant difference(L.S.D) at 5\%level of significant was used. according to (Snedecor and Cochran 1980).

\section{Results and Discussion}

\section{1- Corn hybrids :-}

Available results in Tables ( $1 \mathrm{a}, \mathrm{b}$ and $2 \mathrm{a}, \mathrm{b})$ explain that the main effect of corn hybrids had marked impact on yield characters and chemical content. Planting Single hybrid $30 \mathrm{~K}$ 8(SH30k8) led to significantly increased all yield and chemical characters studied i.e. plant height $(\mathrm{cm})$, ear length (cm), ear diameters (cm), no. of grains/ear, 100- grain weight ( $\mathrm{g})$, grain and biological yields ( $\mathrm{kg} / \mathrm{fed})$, $\mathrm{N}(\%), \mathrm{P}(\%), \mathrm{K}(\%)$ and crud protein content( \%) (Table 1a,b) except, no. of ear /plant by High Tech Hybrid( HTH) and stover yield (kg/ fed) and total carbohydrate (\%) were on Triangle Hybrid (TH) in the first growing season. In the second season, planting Single hybrid $30 \mathrm{~K} 8(\mathrm{SH} 30 \mathrm{k} 8)$ led to significantly increased all yield and chemical characters studied. Similar results were reported by. The differences between hybrids may be due to temperature and Relative humidity at region. Similar results were obtained by (Wolietal., 2016) he found that, High night time temperatures cause plants to expend unnecessary energy (carbohydrate) to maintain plant functions. Those carbohydrates would normally be moved to the ear for storage. Sensing the lack of energy available, the plant respondsby reducing the number of kernels it will attempt to fill. single hybrid $30 \mathrm{~K} 8$ superiority may be due to favorable weather conditions under this region.Mean temperature of April, May, Jun, July at Al frafra region were $37.90,39.24,40.65,43.74$ and 37.12 , $38.89,41.25,44.25$ in both seasons respectively. Therefore, Relative humidity, ofApril, May, Jun, July were 60.44, 59.29,58.23,56.41 and $61.84,60.80,59.22,57.18$ in the first and second seasons respectively 
Table 1a. Effect of corn hybrids on yield, its components in 2014 and 2015 seasons under AL-Frafra Oasis.

\begin{tabular}{|c|c|c|c|c|c|c|c|c|c|}
\hline Characters & $\begin{array}{c}\text { Plant } \\
\text { height } \\
(\mathbf{c m})\end{array}$ & $\begin{array}{c}\text { Ear } \\
\text { length } \\
(\mathrm{cm})\end{array}$ & $\begin{array}{c}\text { Ear } \\
\text { diameters } \\
(\mathbf{c m})\end{array}$ & $\begin{array}{c}\text { No. of } \\
\text { ear } \\
\text { /plant }\end{array}$ & $\begin{array}{c}\text { No. of } \\
\text { grains } \\
\text { / ear }\end{array}$ & $\begin{array}{c}\text { 100- } \\
\text { grains } \\
\text { weight } \\
\text { (g.) }\end{array}$ & $\begin{array}{c}\text { Grain } \\
\text { yield } \\
\text { (kg } \\
\text { /fed) }\end{array}$ & $\begin{array}{c}\text { Stover } \\
\text { yield } \\
\text { (kg } \\
\text { /fed) }\end{array}$ & $\begin{array}{c}\text { Biological } \\
\text { Yield } \\
\text { (kg/fed.) }\end{array}$ \\
\hline \multicolumn{10}{|c|}{2014} \\
\hline (HTH) & 224.37 & 25.86 & 14.80 & 1.45 & 126.79 & 30.58 & 1075.90 & 759.12 & 1835.03 \\
\hline (SH30k8) & 237.50 & 27.01 & 15.18 & 1.43 & 145.75 & 32.20 & 1376.99 & 899.87 & 2276.87 \\
\hline (TH) & 226.87 & 25.47 & 14.53 & 1.42 & 117.83 & 29.21 & 1004.66 & 1025.58 & 2030.25 \\
\hline $\operatorname{LSD}(0.05)$ & 9.021 & 1.251 & 0.61 & 0.013 & 7.13 & 0.48 & 63.13 & 43.12 & 113.24 \\
\hline \multicolumn{10}{|c|}{2015} \\
\hline (HTH) & 204.95 & 27.06 & 13.67 & 1.34 & 148.33 & 30.11 & 1198.49 & 825.58 & 2024.07 \\
\hline (SH30k8) & 230.37 & 29.05 & 13.71 & 1.40 & 157.12 & 31.25 & 1409.18 & 912.79 & 2321.97 \\
\hline (TH) & 211.79 & 23.93 & 13.28 & 1.37 & 137.66 & 30.12 & 1109.98 & 844.33 & 1954.34 \\
\hline $\operatorname{LSD}(0.05)$ & 8.12 & 1.31 & 0.39 & 0.021 & 8.17 & 0.43 & 68.61 & 51.61 & 117.83 \\
\hline
\end{tabular}

Table 1b. Effect of corn hybrids on grains chemical contents in 2014 and 2015 seasons under AL-Frafra Oasis

\begin{tabular}{|c|c|c|c|c|c|}
\hline $\begin{array}{l}\text { Characters } \\
(\mathrm{WCH}) \\
\end{array}$ & $\begin{array}{c}N \\
(\%)\end{array}$ & $\begin{array}{l}\mathbf{P} \\
(\%)\end{array}$ & $\begin{array}{c}\mathrm{K} \\
(\%)\end{array}$ & $\begin{array}{c}\text { Crud protein } \\
(\%)\end{array}$ & $\begin{array}{c}\text { Total } \\
\text { carbohydrate } \\
(\%) \\
\end{array}$ \\
\hline \multicolumn{6}{|c|}{2014} \\
\hline (HTH) & 1.657 & 0.219 & 0.357 & 10.36 & 80.538 \\
\hline (SH30k8) & 1.737 & 0.231 & 0.374 & 10.86 & 80.693 \\
\hline (TH) & 1.705 & 0.221 & 0.321 & 10.56 & 80.595 \\
\hline $\operatorname{LSD}(0.05)$ & 0.051 & 0.011 & 0.031 & 0.31 & 0.030 \\
\hline \multicolumn{6}{|c|}{2015} \\
\hline (HTH) & 1.726 & 0.245 & 0.349 & 10.79 & 78.751 \\
\hline (SH30k8) & 1.745 & 0.236 & 0.367 & 10.91 & 79.046 \\
\hline (TH) & 1.668 & 0.215 & 0.332 & 10.43 & 77.628 \\
\hline $\operatorname{LSD}(0.05)$ & 0.027 & 0.012 & 0.029 & 0.030 & 0.013 \\
\hline
\end{tabular}

$\mathrm{WCH}=$ white corn hybrids, HTH =High Tech Hybrid, SH30k8= Single hybrid $30 \mathrm{~K} 8$, TH= Triangle Hybrid.

\section{Bio-Mineral Fertilizers:}

Tables $(2 a, b)$ indicate that the effect of Biomineral fertilizers on yield and its components , Adding F6 significantly increased plant height $(\mathrm{cm})$, ear length $(\mathrm{cm})$, ear diameters $(\mathrm{cm})$, no. of ear/plant, no. of grains/ear, 100- grains weight ( $\mathrm{g})$, grain and biological yields (kg/fed), $\mathrm{K}(\%)$ and total carbohydrate ( $\%$ )while $100 \%$ mineral fertilizer increased criteria i.e. stover yield, $\mathrm{N}(\%)$ and crud proteinand the highest $\mathrm{P}(\%)$ was $\mathrm{F} 3$ in the first. In the second season, the highest values of all studied parameters increased by F6 except, 100- grains weight , N, K and crud protein were obtained by F2, while $\mathrm{P}(\%)$ was obtained by F3.On the other hand, the insignificantly increased between F2 and F6 to ear length, no. of ear/plant, no. of grains/ear, grain, stover yields, also not significantly different among levels of F6 and F7 bio- mineral fertilization of ear length, no. of ear/plant, no. of grains/ear stover yield in the first season compared with the control treatment. Similarly, F6 significantly affected plant height, ear length, ear diameters, no. of ear/plant, no. of grains/ear grain yield, biological yield, $\mathrm{P}$ and $\mathrm{K}$, while 100 grains weight, grain yield, $\mathrm{N}(\%)$, crud protein in content and totalcarbohydrate increased significantly by F2 in the second season However, F6 had not significantamong $\mathrm{F} 2$ and F5 in most parameters in both seasons compared with the control treatment. Similar results were obtained in the second season. The un lower treatment control got the least of yield and its components.Bio- fertilizer can be used as supplemental to improve and increase the yield of corn; A follow-up study be conducted to evaluate the effect of the different fertilizers during the early season (April to June).yield and its components.

Corn was sown as early summer seasons ( $1^{\text {st }}$ April to $30^{\text {nd }}$ June ) (see material\& method), So corn plants act as F6. F6 there was no significant difference between them and F2 and F6 in most previous parameters..(Rajendraet al.,1998;Hegdeet al., 1999; Vessey 2003 and Khaliqet al., 2006) reported that the microorganisms and the enzymes in these different fertilizers had a significant effect on the yield of the corn plants, the demographic growth explosion and increased in nutritional demand, and 
where agricultural productivity is decreasing at a rate of $0.4 \%$ (Anon, 2009). Hence, local measures and new strategies can improve soil fertility,while maintaining the diversity of cultures and reducing chemical fertilizer uses. Humic acids isolated from earthworm compost enhance root elongation, lateral root emergence and plasma membrane H+-ATPas activity in maize roots (Canellas et al., 2000). The increasing of yield and its components may be due to a bio-fertilizer is a substance which contains living microorganisms which, when applied to seed, plant surfaces, or soil, colonizes the Rhizosphere or the interior of the plant and promotes growth by increasing the supply or availability of primary nutrients to the host plant.

Bio-fertilizers add nutrients through the natural processes of nitrogen, fixation, solubilizing phosphorus, and stimulating plant growth through the synthesis of growth-promoting substances. Biofertilizers can be expected to reduce the use of chemical fertilizers and pesticides. The microorganisms in bio-fertilizers restore the soils natural nutrient cycle and build soil organic matter. Through the use of bio-fertilizers, healthy plants can be grown, while enhancing the sustainability and the health of the soil. Since they play several roles, a preferred scientific term for such beneficial bacteria is "plant-growth promoting rhizobacteria" (PGPR). Therefore, they are extremely advantageous in enriching soil fertility and fulfilling plant nutrient requirements by supplying the organic nutrients through microorganism and their byproducts. Hence, bio-fertilizersdonot contain any chemicals which are harmful to the living soil, Wikipedia, (2014).

Table 2a. Effect of bio-mineral fertilizers on yield, its Components and chemical contents of corn in $2014-$ 2015 seasons.

\begin{tabular}{|c|c|c|c|c|c|c|c|c|c|}
\hline Characters & $\begin{array}{c}\text { Plant } \\
\text { height } \\
(\mathrm{cm})\end{array}$ & $\begin{array}{c}\text { Ear } \\
\text { length } \\
(\mathrm{cm})\end{array}$ & $\begin{array}{c}\text { Ear } \\
\text { diameters } \\
(\mathrm{cm})\end{array}$ & $\begin{array}{c}\text { No. of } \\
\text { ear } \\
\text { /plant }\end{array}$ & $\begin{array}{l}\text { No. of } \\
\text { grains } \\
\text { / ear }\end{array}$ & $\begin{array}{c}\text { 100- } \\
\text { grains } \\
\text { weight } \\
\text { (g.) }\end{array}$ & $\begin{array}{c}\text { Grain } \\
\text { yield } \\
\text { (kg } \\
\text { /fed.) }\end{array}$ & $\begin{array}{c}\text { Stover } \\
\text { yield } \\
\text { (Kg/fed.) }\end{array}$ & $\begin{array}{c}\text { Biological } \\
\text { Yield } \\
\text { (kg/fed.) }\end{array}$ \\
\hline \multicolumn{10}{|c|}{2014} \\
\hline F1 without & 144.88 & 23.78 & 13.73 & 1.17 & 65.66 & 28.74 & 417.63 & 627.11 & 1044.74 \\
\hline F2( $100 \%$ & 225.66 & 27.50 & 15.53 & 1.57 & 133.77 & 30.26 & $\mathbf{1 1 6 4 . 3 2}$ & 1055.55 & 2219.88 \\
\hline F3(Riz. +75) & 255.55 & 27.13 & 15.40 & 1.52 & 156.44 & 31.94 & $\mathbf{1 3 3 4 . 8 2}$ & 997.66 & 2332.48 \\
\hline F4(Riz. + +50) & 250.22 & 25.44 & 14.56 & 1.53 & 151.01 & 31.32 & 586.66 & 755.44 & 2180.64 \\
\hline F5(Riz. +25) & 221.77 & 24.60 & 14.43 & 1.38 & 86.22 & 31.64 & 713.67 & 586.63 & 1967.22 \\
\hline F6(Az. +75) & 269.33 & 28.30 & 15.86 & 1.54 & 170.33 & 32.30 & 1773.04 & 993.33 & 2766.23 \\
\hline F7(Az. + 50) & 256.44 & 26.52 & 15.10 & 1.44 & 164.33 & 30.93 & 1647.96 & 778.33 & 2326.29 \\
\hline F8(Az. +25) & 212.77 & 25.48 & 14.10 & 1.28 & 105.23 & 28.78 & 643.53 & 697.88 & 1341.41 \\
\hline LSD(0.05) & 10.61 & 1.32 & 1.12 & 0.27 & 8.93 & 0.43 & 73.31 & 69.63 & 93.46 \\
\hline \multicolumn{10}{|c|}{2015} \\
\hline F1 without & 132.77 & 21.6 & 12.10 & 1.14 & 70.44 & 27.81 & 411.87 & 669.55 & 1081.42 \\
\hline F2( $100 \%$ & 218.11 & 26.19 & 14.10 & 1.27 & 149.22 & 33.61 & $\mathbf{1 1 4 9 . 8 4}$ & $\mathbf{1 1 0 7 . 8 8}$ & 2257.73 \\
\hline F3(Riz. +75) & 246.01 & 27.13 & 14.26 & 1.53 & 189.77 & 31.37 & $\mathbf{1 8 1 2 . 7 1}$ & 1054.11 & 2866.83 \\
\hline F4(Riz. +50) & 246.11 & 25.21 & 13.43 & 1.51 & 177.33 & 29.04 & 1565.13 & 836.77 & 2401.91 \\
\hline F5(Riz. +25) & 216.11 & 23.25 & 13.03 & 1.27 & 96.88 & 30.46 & 730.41 & 686.77 & 1417.19 \\
\hline F6(Az. +75) & 252.11 & 29.43 & 14.43 & 1.56 & 191.11 & 32.45 & 1963.68 & 947.99 & 2871.68 \\
\hline F7(Az. +50) & 214.77 & 28.24 & 13.70 & 1.45 & 183.66 & 30.46 & 1539.47 & 905.22 & 2484.69 \\
\hline F8(Az. +25) & 199.66 & 25.47 & 13.40 & 1.18 & 116.22 & 28.73 & 740.60 & 678.88 & 1419.49 \\
\hline $\operatorname{LSD}(0.05)$ & 9.73 & 1.03 & 0.93 & 0.19 & 7.13 & 0.34 & 81.21 & 71.81 & 96.31 \\
\hline
\end{tabular}


Table 2b. Effect of bio-mineral fertilizers on chemical contents of corn grains in $2014-2015$ seasons under AL-Frafra Oasis.

\begin{tabular}{|c|c|c|c|c|c|}
\hline Characters & $\begin{array}{c}\mathrm{N} \\
(\%)\end{array}$ & $\begin{array}{c}\mathbf{P} \\
(\%)\end{array}$ & $\underset{(\%)}{K}$ & $\begin{array}{c}\text { Crud protein } \\
(\%)\end{array}$ & $\begin{array}{c}\text { Total } \\
\text { Carbohydrate }(\%)\end{array}$ \\
\hline \multicolumn{6}{|c|}{2014} \\
\hline F1 without (control) & 1.516 & 0.203 & 0.332 & 9.48 & 78.240 \\
\hline F2( $100 \%$ mineral $)$ & 1.819 & 0.229 & 0.377 & 11.37 & 79.247 \\
\hline F3(Riz. +75) & 1.795 & 0.260 & 0.367 & 11.22 & 80.214 \\
\hline F4(Riz. +50) & 1.768 & 0.224 & 0.351 & 11.05 & 80.227 \\
\hline F5(Riz. +25 ) & 1.662 & 0.214 & 0.345 & 10.39 & 79.381 \\
\hline F6(Az. +75) & 1.750 & 0.237 & 0.370 & 10.94 & 80.668 \\
\hline F7(Az. +50) & 1.678 & 0.216 & 0.353 & 10.49 & 80.521 \\
\hline F8(Az. +25) & 1.611 & 0.207 & 0.339 & 10.07 & 79.631 \\
\hline $\operatorname{LSD}(0.05)$ & 0.081 & 0.013 & 0.041 & 0.17 & 0.020 \\
\hline \multicolumn{6}{|c|}{2015} \\
\hline F1 without (control) & 1.460 & 0.201 & 0.333 & 9.13 & 77.346 \\
\hline F2( $100 \%$ mineral) & 1.833 & 0.231 & 0.381 & 11.46 & 78.470 \\
\hline F3(Riz. +75) & 1.798 & 0.273 & 0.369 & 11.24 & 79.096 \\
\hline F4(Riz. +50) & 1.724 & 0.253 & 0.353 & 10.78 & $\mathbf{7 8 . 7 8 0}$ \\
\hline F5(Riz. +25 ) & 1.616 & 0.233 & 0.345 & 10.10 & 77.776 \\
\hline F6(Az. +75) & 1.816 & 0.251 & 0.371 & 11.35 & 79.656 \\
\hline F7(Az. +50) & 1.790 & 0.232 & 0.355 & 11.19 & 78.876 \\
\hline F8(Az. +25) & 1.668 & 0.211 & 0.341 & 10.43 & 77.810 \\
\hline $\operatorname{LSD}(0.05)$ & 0.049 & 0.062 & $\mathbf{0 . 0 3 7}$ & 0.24 & 0.016 \\
\hline
\end{tabular}

3 - The interaction between corn hybrids and biomineral fertilizers .

Tables ( $3 \mathrm{a}, \mathrm{b}, \mathrm{c}$ and $\mathrm{d}$ ) indicate the effect of the interaction between white corn hybrids and BioMineral Fertilizers application on the yield, its components and chemical contents of corn plant. Data revealed that maximum criteriai.e., ear length, ear diameters, no. of grains/ear, 100 grains weight, grain yield, stover yield, biological yield, $\mathrm{N}(\%), \mathrm{P}$ and crud protein in content, were obtained by Single hybrid $30 \mathrm{~K}$ 8(SH30k8) and F6 (Az.+75\% mineral fertilizer) while plant height, no. of ear/plant, $\mathrm{K}$, and total carbohydrate were obtained by F2 in first season. Similarly, results were obtained in second season except plant height and total carbohydrate obtained by Single hybrid $30 \mathrm{~K} 8$ (SH30k8) and F2 (100\% mineral fertilizer). This is true in both seasons. Several investigators pointed out that yield and its components were increased by using (SH30k8) and F6(PYH-3084) (Elamin, and Elagib 2001;Kuepper 2003; Efthimiadouet al., 2010; Baral and Adhikari 2013; Ghasemiet al., 2013;Beyranvet al., 2013 and Khaksaraet al., 2014).
Economic Importance of corn hybrid.

The experimental inputs and outputs as well as the ratio between outputs and inputs for each treatment introducing investment ratio (IR) under the condition under AL-Frafra Oasis, The results indicated the progressive increment in IR by increasing of corn plants to three white corn hybrids as affected by six levels combination between biofertilization and mineralization. Found from the calculation of the economic yield of crop, yield and its components and chemical contents, the use of Single hybrid $30 \mathrm{~K} 8(\mathrm{SH} 30 \mathrm{k} 8)$ and F6 (Az. $+75 \%$ mineral fertilizer) were the best experimental economic transaction for the farmer under the conditions of AL-Frafra Oasis.

\section{Recommendation:}

It could be concluded that variety of Single hybrid $30 \mathrm{~K} 8$ (SH30k8) was an promising and F6 (Az. +75 $\%$ mineral fertilizer) fertilizer for increasing corn production under AL-Frafra Oasis conditions in Egypt. Based on the above findings, it is highly recommended that the application NPK 75 $\%$ fertilizer combined with Aztobacter -fertilizers in corn be adopted by farmers. To lessen production cost. 
Table 3a. Effect of interactions between corn hybrids and bio-mineralfertilizers on yield and its components in first season 2014 under AL Frafra Oasis.

\begin{tabular}{|c|c|c|c|c|c|c|c|c|c|c|}
\hline \multicolumn{2}{|c|}{$\mathbf{W C H} \times \mathbf{B M F}$} & $\begin{array}{c}\text { Plant } \\
\text { height } \\
(\mathbf{c m} .)\end{array}$ & $\begin{array}{l}\text { Ear length } \\
\quad(\mathrm{cm} .)\end{array}$ & $\begin{array}{c}\text { Ear } \\
\text { diam- } \\
\text { eters } \\
(\mathrm{cm} .)\end{array}$ & $\begin{array}{l}\text { No. of ear/ } \\
\text { plant }\end{array}$ & $\begin{array}{l}\text { No. of } \\
\text { grains / } \\
\text { ear }\end{array}$ & $\begin{array}{l}\text { 100- grains } \\
\text { weight (g.) }\end{array}$ & $\begin{array}{c}\text { Grain yield } \\
\text { (kg /fed.) }\end{array}$ & $\begin{array}{c}\text { Stover yield } \\
\text { (Kg/fed.) }\end{array}$ & $\begin{array}{c}\text { Biological } \\
\text { Yield } \\
\text { (kg/fed.) }\end{array}$ \\
\hline \multicolumn{11}{|c|}{2014} \\
\hline \multirow{8}{*}{ HTH } & F1 without (control) & 125.0 & 23.2 & 13.5 & 1.13 & 57.66 & 29.46 & 365.03 & 633.6 & 998.7 \\
\hline & F2( $100 \%$ mineral $)$ & 241.3 & 27.6 & 15.5 & 1.56 & 128.66 & 30.23 & 1194.927 & 1056.0 & 2250.9 \\
\hline & F3(Riz. +75) & 243.0 & 27.3 & 15.4 & 1.56 & 151.66 & 31.26 & 1435.933 & 1010.3 & 2446.2 \\
\hline & F4(Riz. +50) & 243.3 & 25.3 & 14.5 & 1.56 & 146.33 & 31.53 & 1386.953 & 726.6 & 2113.6 \\
\hline & F5(Riz. + 25) & 243.6 & 24.7 & 14.4 & 1.30 & 85.33 & 30.26 & 638.82 & 637.0 & 1275.8 \\
\hline & F6(Az. +75) & 254.0 & 28.5 & 15.9 & 1.50 & 176.33 & 32.33 & 1730.767 & 937.6 & 2668.4 \\
\hline & F7(Az. + +50) & 242.6 & 24.7 & 15.1 & 1.56 & 165.66 & 30.23 & 1527.88 & 393.3 & 1921.2 \\
\hline & F8(Az. +25) & 202.0 & 25.5 & 14.1 & 1.30 & 102.66 & 29.36 & 326.94 & 678.3 & 1005.2 \\
\hline \multirow{8}{*}{ SH30k8 } & F1 without (control) & 142.33 & 25.5 & 14.4 & 1.33 & 74.33 & 29.46 & 533.6 & 663.6 & 1197.0 \\
\hline & F2( $100 \%$ mineral $)$ & 262.0 & 28.3 & 15.9 & 1.63 & 156.33 & 32.13 & 1252.767 & 1092.3 & 2345.1 \\
\hline & F3(Riz. +75) & 258.0 & 27.4 & 15.6 & 1.50 & 181.66 & 33.30 & 1573.10 & 1004.3 & 2577.4 \\
\hline & F4(Riz. +50) & 252.3 & 26.5 & 14.9 & 1.56 & 164.66 & 32.20 & 1596.50 & 862.3 & 2458.8 \\
\hline & F5(Riz. + 25) & 255.6 & 25.5 & 14.7 & 1.56 & 97.00 & 35.46 & 928.25 & 724.6 & 1652.9 \\
\hline & F6(Az. +75) & 253.6 & 28.6 & 16.3 & 1.46 & 195.01 & 34.30 & 2094.04 & 1098.3 & 3162.3 \\
\hline & F7 $(A z .+50)$ & 261.6 & 27.5 & 15.4 & 1.26 & 181.10 & 33.20 & 2093.88 & 1011.6 & 3160.5 \\
\hline & F8(Az. + 25$)$ & 214.3 & 26.5 & 14.3 & 1.26 & 116.02 & 27.53 & 949.07 & 771.6 & 1720.7 \\
\hline \multirow{8}{*}{ TH } & F1 without (control) & 127.3 & 22.6 & 13.3 & 1.06 & 65.03 & 27.30 & 354.51 & $\mathbf{5 8 4 . 0}$ & 938.5 \\
\hline & F2( $100 \%$ mineral $)$ & 173.6 & 26.5 & 15.2 & 1.56 & 116.33 & 28.43 & 1045.28 & 1018.3 & 2063.6 \\
\hline & F3(Riz. +75) & 265.6 & 26.6 & 15.2 & 1.50 & 136.04 & 29.43 & 1295.42 & 978.3 & 2273.7 \\
\hline & F4(Riz. +50) & 254.0 & 24.4 & 14.3 & 1.60 & 142.01 & 30.23 & 1292.15 & 677.3 & 1969.4 \\
\hline & F5(Riz. +25 ) & 155.0 & 23.5 & 14.2 & 1.30 & 76.33 & 29.20 & 573.94 & 399.0 & 2972.9 \\
\hline & F6 $($ Az. + 75) & 300.3 & 27.7 & 15.4 & 1.56 & 163.66 & 30.26 & 1494.33 & 974.0 & 2468.3 \\
\hline & F7(Az. +50) & 265.0 & 27.3 & 14.8 & 1.50 & 146.33 & 29.36 & 1327.13 & 930.0 & 2257.1 \\
\hline & F8(Az. +25) & 222.0 & 24.4 & 13.9 & 1.30 & 97.01 & 29.46 & 654.56 & 643.6 & 1298.2 \\
\hline $\operatorname{LSD}(0.05)$ & & 1.53 & 0.21 & 0.41 & 0.12 & 0.51 & 0.91 & 0.21 & 0.51 & 2.91 \\
\hline
\end{tabular}


Table 3b. Effect of interactions between corn hybrids and bio-mineral fertilizers on chemical contents in first season 2014 under AL Frafra Oasis.

\begin{tabular}{|c|c|c|c|c|c|c|}
\hline \multicolumn{2}{|l|}{$\begin{array}{l}\text { Characters } \\
\mathbf{W C H} \times \mathbf{B M F}\end{array}$} & $\begin{array}{c}\mathrm{N} \\
(\%)\end{array}$ & $\begin{array}{c}P \\
(\%)\end{array}$ & $\begin{array}{c}\mathrm{K} \\
(\%)\end{array}$ & $\begin{array}{c}\text { Crud } \\
\text { Protein } \\
(\%)\end{array}$ & $\begin{array}{c}\text { Total } \\
\text { carbohydrate } \\
(\%)\end{array}$ \\
\hline \multicolumn{7}{|c|}{2014} \\
\hline \multirow{8}{*}{ HTH } & F1 without (control) & 1.489 & 0.201 & 0.331 & 9.32 & 78.150 \\
\hline & F2( $100 \%$ mineral $)$ & 1.756 & 0.226 & 0.368 & 10.98 & 79.200 \\
\hline & F3(Riz. +75) & 1.731 & 0.258 & 0.359 & 10.82 & 80.012 \\
\hline & F4(Riz. +50) & 1.716 & 0.215 & 0.341 & 10.73 & 80.271 \\
\hline & F5(Riz. +25 ) & 1.608 & 0.211 & 0.338 & 10.05 & 79.320 \\
\hline & F6(Az. +75) & 1.697 & 0.218 & 0.361 & 10.61 & 80.263 \\
\hline & F7(Az. +50) & 1.649 & 0.217 & 0.344 & 10.31 & 80.521 \\
\hline & F8(Az. +25) & 1.612 & 0.209 & 0.340 & 10.08 & 79.823 \\
\hline \multirow{8}{*}{ SH30k8 } & F1 without (control) & 1.521 & 0.207 & $\mathbf{0 . 3 3 7}$ & 9.51 & 79.220 \\
\hline & F2( $100 \%$ mineral $)$ & 1.905 & 0.229 & 0.389 & 11.91 & 80.120 \\
\hline & F3(Riz. +75) & 1.875 & 0.264 & 0.381 & 11.72 & 81.312 \\
\hline & F4(Riz. +50) & 1.825 & 0.252 & 0.362 & 11.41 & 81.171 \\
\hline & F5(Riz. +25 ) & 1.697 & 0.231 & 0.350 & 10.61 & 80.231 \\
\hline & F6(Az. +75) & 1.793 & 0.234 & 0.385 & 11.21 & 81.321 \\
\hline & F7(Az. +50) & 1.686 & 0.221 & 0.366 & 10.54 & 81.110 \\
\hline & F8(Az. +25$)$ & 1.603 & 0.213 & 0.348 & 10.02 & 80.751 \\
\hline \multirow{8}{*}{ TH } & F1 without (control) & 1.539 & 0.202 & 0.328 & 9.62 & 77.351 \\
\hline & F2( $100 \%$ mineral $)$ & 1.796 & 0.234 & 0.376 & 11.23 & 78.421 \\
\hline & F3(Riz. +75) & 1.779 & 0.259 & 0.363 & 11.12 & 79.320 \\
\hline & F4(Riz. +50) & 1.763 & 0.206 & 0.350 & 11.02 & 79.241 \\
\hline & F5(Riz. +25) & 1.684 & 0.201 & 0.348 & 10.53 & 78.592 \\
\hline & F6(Az. +75) & 1.761 & 0.261 & 0.366 & 11.01 & 80.421 \\
\hline & F7(Az. +50) & 1.699 & 0.211 & 0.349 & 10.62 & 79.932 \\
\hline & F8(Az. +25) & 1.620 & 0.201 & 0.331 & 10.13 & 78.320 \\
\hline $\operatorname{LSD}(0.05)$ & & 0.0198 & $\mathbf{0 . 0 2 3}$ & 0.041 & 0.011 & 0.090 \\
\hline
\end{tabular}


Table 3c. Effect of interactions between corn hybrids and bio-mineral fertilizers on yield and its components in second season 2015 under AL Frafra Oasis.

\begin{tabular}{|c|c|c|c|c|c|c|c|c|c|c|}
\hline $\mathbf{W C H} \times \mathbf{B M}$ & Characters & $\begin{array}{c}\text { Plant } \\
\text { height } \\
(\mathrm{cm})\end{array}$ & $\begin{array}{l}\text { Ear } \\
\text { length } \\
(\mathrm{cm})\end{array}$ & $\begin{array}{c}\text { Ear } \\
\text { diameters } \\
\mathbf{c m})\end{array}$ & $\begin{array}{c}\text { No .of } \\
\text { ear } \\
\text { /plant }\end{array}$ & $\begin{array}{l}\text { No. of } \\
\text { grains / } \\
\text { ear }\end{array}$ & $\begin{array}{l}\text { 100- grains } \\
\text { weight (g) }\end{array}$ & $\begin{array}{c}\text { Grain } \\
\text { yield } \\
(\mathrm{kg} / \mathrm{fed})\end{array}$ & $\begin{array}{c}\text { Stover } \\
\text { yield } \\
\text { (kg /fed) }\end{array}$ & $\begin{array}{c}\text { Biological } \\
\text { Yield } \\
\text { (kg /fed) }\end{array}$ \\
\hline \multicolumn{11}{|c|}{2015} \\
\hline \multirow{6}{*}{ HTH } & F1 without (control) & 117.3 & 22.5 & 12.2 & 1.13 & 66.0 & 27.60 & 384.33 & 650.01 & 1034.33 \\
\hline & F2( $100 \%$ mineral $)$ & 234.0 & 26.4 & 14.5 & 1.26 & 143.6 & 31.83 & 1017.067 & 1092.66 & 2109.73 \\
\hline & F3(Riz. +75) & 237.0 & 28.5 & 14.2 & 1.56 & 191.3 & 31.50 & 1815.413 & 1018.01 & 2880.41 \\
\hline & F4(Riz. + +50) & 245.0 & 26.5 & 13.4 & 1.50 & 183.3 & 28.33 & 1575.043 & 839.66 & 2414.71 \\
\hline & F7(Az. +50) & 136.3 & 27.4 & 13.8 & 1.26 & 185.0 & 29.33 & 1346.573 & 857.33 & 2203.90 \\
\hline & F8(Az. +25) & 187.0 & 26.4 & 13.5 & 1.13 & 115.0 & 28.20 & 689.11 & 720.04 & 1409.11 \\
\hline \multirow{5}{*}{ SH30k8 } & F1 without (control) & 134.6 & 21.6 & 12.3 & 1.16 & 77.3 & 28.40 & 458.67 & 717.33 & 1176.00 \\
\hline & F2( 100\% mineral) & 256.0 & 27.5 & 14.3 & 1.30 & 156.6 & 33.66 & 1292.013 & 1143.03 & 2435.01 \\
\hline & F3(Riz. +75) & 246.6 & 27.5 & 14.5 & 1.55 & 202.0 & 32.26 & 1970.74 & 1034.66 & 3005.40 \\
\hline & F4(Riz. + 50) & 247.6 & 25.5 & 13.7 & 1.53 & 186.0 & 31.26 & 1762.49 & 908.02 & 2670.49 \\
\hline & F5(Riz. +25) & 254.3 & 23.6 & 13.3 & 1.26 & 107.0 & 29.36 & 795.80 & 689.33 & 1485.14 \\
\hline \multirow{6}{*}{ TH } & F3(Riz. +75) & 254.3 & 25.4 & 14.1 & 1.56 & 176.0 & 30.36 & 1652.00 & 1062.66 & 2714.67 \\
\hline & F4(Riz. +50) & 245.6 & 23.6 & 13.2 & 1.53 & 162.6 & 27.53 & 1357.88 & 762.66 & 2120.54 \\
\hline & F5(Riz. +25) & 157.0 & 21.6 & 12.7 & 1.26 & 86.6 & 30.46 & 639.86 & 651.33 & 1291.20 \\
\hline & F6(Az. +75) & 255.3 & 26.4 & 14.5 & 1.56 & 181.6 & 31.53 & 1750.13 & 1045.33 & 2795.46 \\
\hline & F7(Az. +50) & 254.3 & 26.6 & 13.4 & 1.56 & 172.3 & 30.70 & 1368.42 & 919.01 & 2287.47 \\
\hline & F8(Az. +25$)$ & 217.0 & 22.5 & 13.1 & 1.13 & 106.6 & 27.60 & 578.49 & 584.33 & 1162.82 \\
\hline $\operatorname{LSD}(0.05)$ & & 0.51 & 0.30 & 0.32 & 0.24 & 0.61 & 0.97 & 0.24 & 0.55 & 1.31 \\
\hline
\end{tabular}


Table 3d. Effect of interactions between corn hybrids and bio-mineral fertilizers on chemical contents in second season 2015 under AL Frafra Oasis.

$$
\text { Characters }
$$

$\mathrm{WCH} \times \mathbf{B M F}$

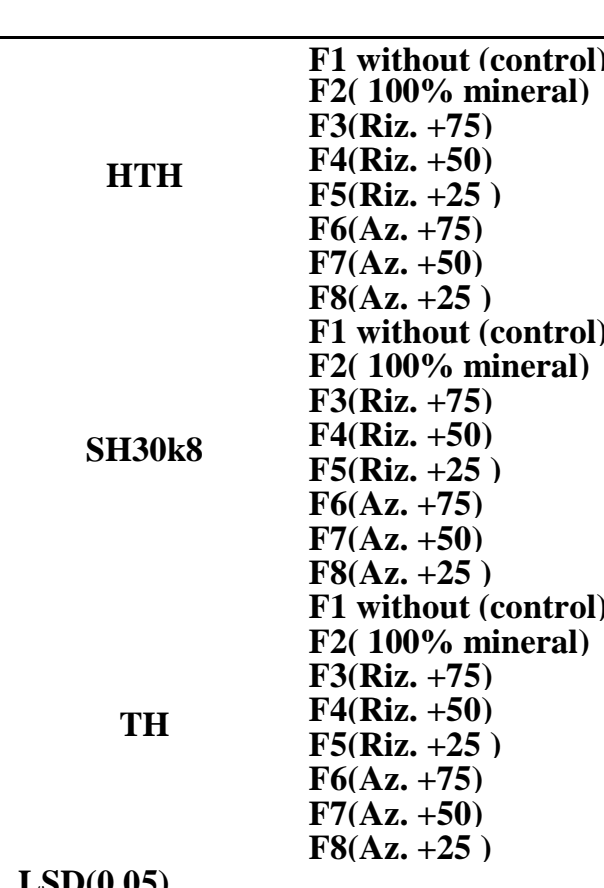

$$
\begin{aligned}
& \mathrm{N} \\
& (\%)
\end{aligned}
$$

2015

2015

$1.462 \quad 0.201$

1.860

1.841

1.774

1.611

1.788
1.764

1.764
1.731

1.478

1.924

1.843

1.763

1.633

1.892

1.798

1.636

1.448

1.734

1.713

1.636
1.606

1.606
1.768

1.809

1.638

0.024

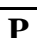

P)

0.224

0.265

0.234

0.215

0.243

0.212

0.212

0.201

0.239

0.272

0.272
0.251

0.251
0.213

0.213
$\mathbf{0 . 2 6 1}$

$\mathbf{0 . 2 6 1}$
$\mathbf{0 . 2 4 2}$

0.213

0.201

0.231

0.231

\begin{tabular}{l}
0.282 \\
\hline 0.276
\end{tabular}

0.276
0.271

$\mathbf{0 . 2 7 1}$
$\mathbf{0 . 2 5 1}$

0.242

0.242

\begin{tabular}{l}
$\mathbf{0 . 0 3 6}$ \\
\hline
\end{tabular}
K

K

Crud Protein

(\%)

Total

carbohydrate

(\%)

77.34

0.33

$\mathbf{0 . 3 3 2}$
$\mathbf{0 . 3 7 2}$

0.372
0.361

$\mathbf{0 . 3 6 1}$

0.339

0.363

0.347

$\mathbf{0 . 3 3 9}$

0.338

0.342

0.383

0.364

0.351

0.391

0.369

0.352

0.331

0.379

0.364

0.351

$\mathbf{0 . 3 4 7}$

0.365

0.350

$\mathbf{0 . 3 2 2}$

0.047

9.14

$\mathbf{9 . 1 4}$

11.51

$\mathbf{7 8 . 5 2}$

$11.51-79.32$

$11.09-79.35$

10.07

$\begin{array}{ll}10.07 & 78.19 \\ 11.18 & 79.53\end{array}$

$\begin{array}{ll}11.03 & 79.15 \\ 10.82 & 78.61\end{array}$

$\begin{array}{ll}10.82 & 78.61 \\ 9.24 & \mathbf{7 8 . 1 9}\end{array}$

$\begin{array}{rr}9.24 & 78.19 \\ 12.03 & 79.22\end{array}$

11.52

11.52

$11.02-79.01$

$\begin{array}{ll}10.21 & 78.23 \\ 11.83 & 80.29\end{array}$

$\begin{array}{ll}11.83 & 80.29 \\ 11.24 & 79.43\end{array}$

$\begin{array}{ll}11.24 & 79.43 \\ 10.23 & 78.18\end{array}$

$\begin{array}{ll}11.23 & 78.18 \\ 9.05 & \mathbf{7 6 . 5 1}\end{array}$

$\begin{array}{ll}9.05 & 76.51 \\ 10.84 & 77.67\end{array}$

\begin{tabular}{ll}
10.84 & 77.67 \\
10.71 & 78.15 \\
\hline 10.23 & 77.98
\end{tabular}

$\begin{array}{ll}10.23 & 77.98 \\ 10.04 & 76.91\end{array}$

$\begin{array}{ll}10.04 & 76.91 \\ 11.05 & 79.15\end{array}$

$\begin{array}{ll}11.05 & 79.15 \\ 11.31 & 78.05\end{array}$

\begin{tabular}{cc}
11.31 & 78.05 \\
10.24 & 76.61 \\
0.010 & 0.0281 \\
\hline
\end{tabular}




\section{References}

Ahmed A. M.(2009). Yield and quality of maize in response to biofertilizer application. M.Sc. Thesis, Fac. Agric., Assiut Univ. Egypt.

Ahmed, E.A. (2011). Effect of spatial distribution of plant under different watering regimes on the yield and its components of corn (Zea mays L.). M.Sc. Thesis, Fac. Agric., Assiut Univ. Egypt.

Ahmed, R. (1998). Evaluating some maize varieties under different levels of soil and foliar nitrogen fertilization. M.Sc. Thesis, Fac. Agric., Minia Univ., Egypt.

Ali A.; A. Malik; M.A. Choudhry; M. Khaliq and M. Rafique (1999). Effect of various doses of nitrogen on the growth, yield and protein content of two maize genotypes. Pak. J. Biol. Sci, 2(3): 889-89.

Anon (2009). Economic progress enhances development possibilities for Africa, but soil degradatioremain a major problem. Section of Division Earth Warning and Assessment (DEWA). United Nations Environment Program (UNEP). 4p.

Azimi SM; A. Farnia;M. Shaban, M. Lak (2013a). Effect of different biofertilizers on Seed yield of barley (Hurdeom)

Azimi SM; E. Nabati; M. Shaban and M Lak (2013b).Effect of $\mathrm{N}$ and $\mathrm{P}$ bio fertilizers on yield components of barley.International $\mathrm{j}$. of Advanced Biological and Biomedical Res., 2 (2): 365-370.

Baral B.R, and P. Adhikari (2013). Effect of Azotobacter on Growth and Yield of Maize. SAARC J. Agri; 11(2): 141-147.

Beyranv.H, Farnia A; S .Nakhjavan, and $M$. Shaban (2013). Response of yield and yield components of maize (Zea mayz L.) to different bio fertilizers. International journal of Advanced Biological and Biomedical Res., 1(9): 1068-1077.

Bulalin , (2015): Enhancing corn productivity through application of vermin tea as foliar spray. Asia Pacific Journal of Multidisciplinary Research,. 3, (5): 314- 322.

Canellas, L.P.; F.L. Olivares; A.L. Okorokova and R.A. Facanha(2000). Humic acids isolated from earthworm compost enhance root elongation, lateral root emergence and plasmamembrane $\mathrm{H}+-$ ATPase activity in maize roots. Inter. J. Plant Physiol., 130: 1951-1957.

Chaudhry, A.N.; M.I. Latif; U.R. Haroon; M. Rasheed and J. Ghulam (2005). Profitability increase in maize production through fertilizer management and defoliation under rainfedcropping.International. J. Biol and Biotech. Karachi, Pakistan; 2(4): 1007-1012.

Choudhary, V. K. and P.S. Kumar(2013). Maize production, economics and soil productivity under different organic source of nutrients in eastern himalayan region. India. Int.J. Plant Prod., 7(2): 167-186.

Efthimiadou, A.;D. A. Bilalis; A. Karkanis and B. Froud-Williams (2010). Combined organic/ inorganic fertilization enhance soil quality and increased yield, photosynthesis and sustainability of sweet maize crop. Austr. J. Crop Sci., 4(9):722-729.

Elamin, A.E. and M.A. Elagib(2001). Comparative study of organic and inorganic fertilizers on forage corn (Zea mays L.) grown on two soil types. Qatar Univ. Sci. J., 21: 47-54.

Ghasemi S; M.Ramezani; F.Fatemi-Nik, and A.Bazdar(2013). Effect of microbialphosphate biofertilizer on corn (Zea mays L. S. C. 704) under water deficit stress in the Mehran region of Iran Inter. J. of Agri. Sci.; 3(3): 253-262.

Gomez, K. A. and A. A. Gomez(1984). Statistical Procedures for Agriculture Research. A WileyInter Science Publication, John Wiley \& Sons, Inc. New York, USA.

Hegde D. M.; B. S. Dwived.and S. N.Sudhakara(1999). Biofertilizers for cereal production in India - a review. Indian J. of Agric. Sci., 69(2):73-83.

Ibrahim, S.A and H. Kandil (2007). Growth, Yield and Chemical Constituents of Corn (Zea mays L.) As Affected by Nitrogen and Phosphors Fertilization under Different Irrigation Intervals. J. Appl. Sci. Res., 3(10): 1112-1120.

Johnson C.M. and A. Ulrich.(1959). Analytical methods for use in plant analysis. U.S. Dept. Agric., Calif. Univ., Agric. Inform. Bull., 766.

Khaksara K; $\mathbf{R}$.Chaokanc; H. HeidariSharifabadb; J.Daneshianc;F. .Khazaeiband F.V .Farhadid(2014).Study of grain yield and its components in corn hybrids in two planting dates using different rates of biofertilizers in Karaj region, Iran. Plant Ecophysiology,5 (3): 141-150.

Khaliq A.; M. K . Abbasi and T .Hussain(2006). Effects of integrated use of organic and inorganic nutrient sources with effective microorganisms (EM) on seed cotton yield in Pakistan. Biore. Techno., 97 (8): 967-972.

Khan, M.A.; N.U. Khan; K. Ahmad, M.S. Bloch and M.Sadiq (1999). Yield of maize hybrid-3335 as affected by NP levels. Pak. J. Biol. Sci., 2(3): 857-859.

Kuepper, G. (2003). Manures for Organic Crop Production. Soil System Guide.Appropriate Technology Transfer for Rural Areas (ATTRA), $12 \mathrm{p}$.

Mohamed, E.I.; M.R.A. Hovny and K.A.O. ElAref (2000). Effect of splitting both nitrogenous and fertilizers on Sorghum (Sorghum bicolor L.) productivity and chemical composition.Assiut J. Agric. Sci., Vol 31, No 1, 249-260.

Montogomery R. (1961). Further studies of the phenol sulphoric acid reagent for carbohydrate.Biochem.Biophus. Acta, 48:59. 
Peach K. and M.V. Tracey (1956). Modern methods of plant analysis.Vol. 1.Springer Verlag. Berlin,2, (4): 643.

Rajendra P.; S .Singh., and S.N. Sharma (1998). Inter-relationships of fertilizer use and other agricultural inputs for higher British Biotec. J.; 5(2):102-114.

Sharifi, R.S. and R. Taghizadeh (2009). Response of maize (Zea mays L.) cultivars to different levels of nitrogen fertilizer. J. Food, Agric. and Environ. 7 (3 and 4): 518-521.

Smith D. G.; M. Poulsen and C.A. Raguse(1964). Extraction of total available carbohydrates from grass and legume tissues, Plant physiol., 39: 960.
Snedecor, G.W. and W.G .Cochran(1980). Statistical Methods, 7th.Ed.Iowa State Univ. Press, Ames, Iowa, USA, pp325-330.

Tollenaar, M. and L.M. Dwyer (1999). Physiology of maize. In Smith, D. L. and Hamel, C. (eds). Crop Physiology and Processes. Springer-Verlag, Berlin Heidelberg, pp. 169-199.

Vessey J.K. (2003). Plant growth promoting rhizobacteria as biofertilizers. Plant Soil, Volume 255, Issue 2, pp 571-586.

Woli, K; P. Boyer;J. Matthew; E. R. Wesley;S. John; E. Abendroth;J. Lori and W. Daniel (2016). Corn Era Hybrid Response to Nitrogen Fertilization" Agronomy \& Horticulture -Faculty Publications. 848. 


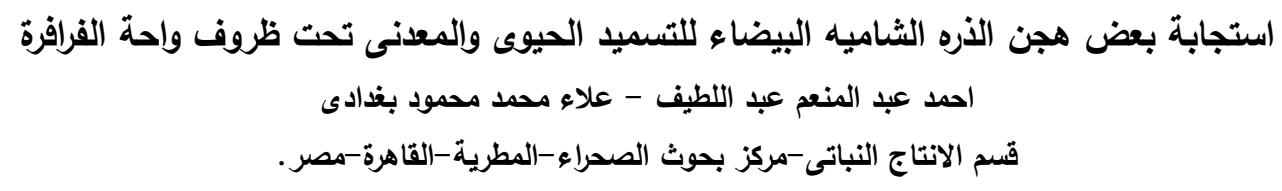

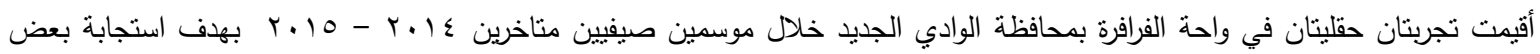

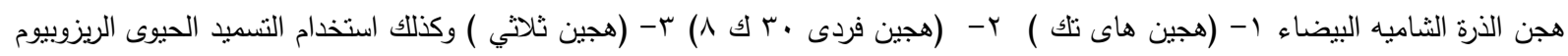

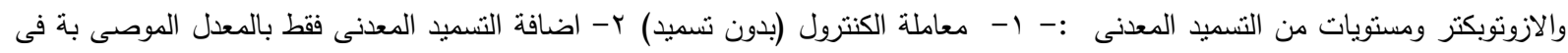

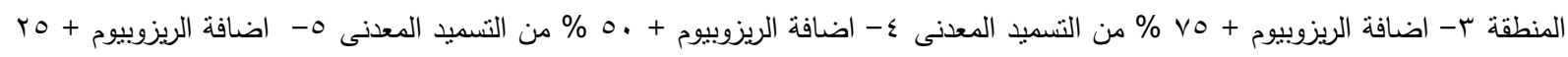

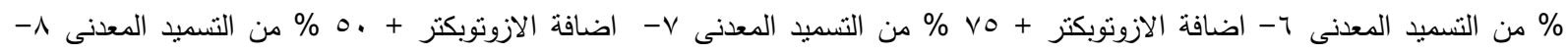

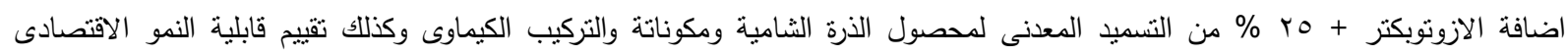

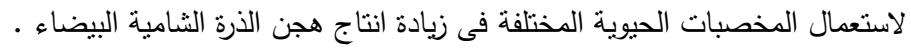

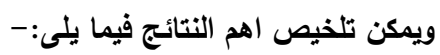
1- كانت هناك زيادة معنوية فى المحصول ومكوناتة وكذلك المحتوى الكيميائى للحبوب وذلك باستخدام صنف هجين فردى • ب كى فى كل من موسمى النمو - ماف ץ- اضافة الازوتوبكتر + • ₹ \% من التسميد المعدنى ادى الى زيادة معنوية فى المحصول ومكوناتة هذا بالاضافة الى زيادة المحتوى الكيميائى

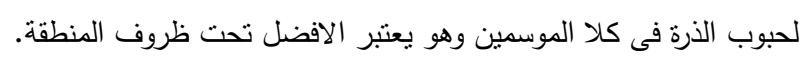
ץ- ادى التفاعل بين اصناف الذرة الثامية ومسنويات التسميد الحيوى الى نأثير معنوى المحصول ومكوناتة. وكانت افضل معاملة هلى هلى التفاعل بين

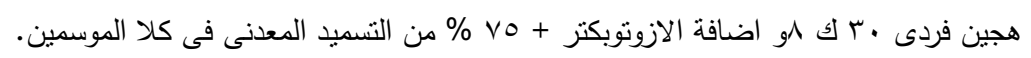

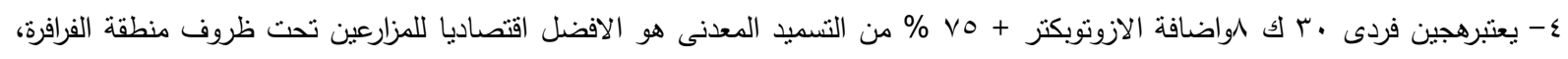

